\title{
The Deliberate Way of Comprehensive Antenatal Care
}

\author{
Panburana $\mathrm{P}^{*}$ \\ Mahidol University, Thailand
}

*Corresponding author: Panyu Panburana, Mahidol University, Thailand, Email:

ppanburana@yahoo.com

\section{Opinion}

Volume 2 Issue 1

Received Date: January 07, 2019

Published Date: January 18, 2019

DOI: $10.23880 /$ jqhe- 16000107

\section{Opinion}

In the past old days all pregnancy care was confined to the time of delivery. This is why there were high mortality and morbidity of both mothers and their babies. The concept of antenatal care was introduced in order to reduce these adverse events during pregnancy. The theory of Developmental Origin of Health and Disease (DOHaD) emphasized the correlation between fetal wellbeing in utero and the adult health and diseases. Eighty years ago, the British ministry of Health recommended the ANC should be followed as in a pyramidal visit. Women should first be seen at 16 weeks, then at 24 and 28 weeks. Thereafter, every fortnight until 36 weeks and then weekly until delivery. Although no explicit rationale was offered for the clinical content of each visit, these pattern remains to be accepted throughout the world even today. Pregnancy management typically involves reacting to maternal and fetal problems only after they develop. Because most fetal and maternal complications become apparent late in pregnancy, it has been traditionally thought that is when the most intensive surveillance should be implemented. Recent decades have seen a movement of fetal and maternal investigations to the first trimester of pregnancy. The idea of the inverted pyramid of prenatal care and monitoring has emerged, for the purpose of prediction and prevention, early detection and treatment of health disorders of the fetus. Firsttrimester ultrasound evaluation provides a very accurate estimation of gestational age as well as the fetal anomalies. The efficiency of screening program of aneuploidy, preeclampsia, gestational diabetes, preterm labor as well as obstructive sleep apnea can estimate patient-specific risks for pregnancy complication in order to improve the pregnancy outcomes. Shifting ANC from routine to more individualized and disease-specific approach both in term of the schedule and content of each visit. Ramathibodi customized ANC has been implemented not only to decrease number of unnecessary visits of low risk pregnancy especially in the third trimester but also to reduce the maternal and fetal mortality and morbidity. Thai NIPT (noninvasive prenatal test) is a detection method of cell free fetal DNA in maternal blood. The whole process is performed at Ramathibodi hospital based on the Thai genome data project. As it is a not-aprofit project, the price has been decreasing each year. Thai NIPT has also integrated into the fetal aneuploidy program in ANC. The cost benefit analysis of Thai NIPT has been published last month indicating that it can be an interesting candidate of fetal aneuploidy model not only in term of its efficiency but also the cost benefit.

\section{Reference}

1. Oraluck P, Boonsong O, Wasun C, Ammarin T, Panyu P (2017) Cost Benefit Analysis of Prenatal Screening Test with Thai NIPT (Thai Non- Invasive Prenatal Test) for Down Syndrome in Developing Countries Health Care Current Reviews 5(3): 207. 